\title{
REFINED YOUNG INEQUALITY WITH KANTOROVICH CONSTANT
}

\author{
Hongliang Zuo, Guanghua Shi And Masatoshi FujiI
}

Abstract. The Specht ratio $S(h)$ is the optimal constant in the reverse of the arithmetic-geometric mean inequality, i.e., if $0<m \leqslant a, b \leqslant M$ and $h=\frac{M}{m}$, then $(1-\mu) a+\mu b \leqslant S(h) a^{1-\mu} b^{\mu}$ for all $\mu \in[0,1]$. Recently S. Furuichi proved that $(1-\mu) a+\mu b \geqslant S\left(h^{r}\right) a^{1-\mu} b^{\mu}$ for $a, b>0$, $\mu \in[0,1]$, where $h=\frac{b}{a}$ and $r=\min \{\mu, 1-\mu\}$. In this paper, we improve it by virtue of the Kantorovich constant, utilizing the refined scalar Young inequality we establish a weighted arithmetic-geometric-harmonic mean inequality for two positive operators. In the remainder of this work we focus on extending the refined weighted arithmetic-harmonic mean inequality to an operator version for another type of improvement.

Mathematics subject classification (2010): 15A45.

Keywords and phrases: Young inequality, Kantorovich constant, Specht ratio, operator inequality, operator means, arithmetic-geometric-harmonic mean inequality.

\section{REFERENCES}

[1] J. BARIĆ, M. Matić, AND J. PeČARIĆ, On the bounds for the normalized Jensen functional and Jensen-Steffensen inequality, Math. Inequal. Appl. 12 (2009), 413-432.

[2] S. FURUICHI, Refined Young inequalities with Specht's ratio, ArXiv:1004. 0581v2.

[3] S. Furuichi, On refined Young inequalities and reverse inequalities, J. Math. Inequal., 5 (2011), $21-31$.

[4] T. FURUTA, The Hölder-McCarthy and the Young inequalities are equivalent for Hilbert space operators, Amer. Math. Monthly 108 (2001), 68-69.

[5] F. Kittaneh And Y. ManasRah, Improved Young and Heinz inequalities for matrices, J. Math. Anal. Appl. 36 (2010), 262-269.

[6] F. Kubo And T. Ando, Means of positive operators, Math. Ann., 264 (1980), 205-224.

[7] D.S. Mitrinović, J. PEČARIĆ AND A.M. FinK, Classical and new inequalities in analysis, Kluwer Academic Publishers, Dordrecht/Boston/London, 1993.

[8] S. Simić, On a new converse of Jensen inequality, Publ. de L'institut Math. 85 (2009), 107-110.

[9] W. SPecht, Zer Theorie der elementaren Mittel, Math. Z. 74 (1960), 91-98.

[10] M. TominaGA, Specht's ratio in the Young inequality, Sci. Math. Japon. 55 (2002), 583-588. 